\title{
Bolus dose of epinephrine for refractory post-arrest hypotension
}

\author{
Michael Gottlieb, MD, RDMS*
}

\begin{abstract}
Post-cardiac arrest hypotension is associated with worse outcomes. However, a significant proportion of patients may not be responsive to intravenous (IV) fluids, and vasopressor infusions require significant time to initiate. This case series describes the successful use of a bolus dose of epinephrine to rapidly treat IV fluid refractory hypotension among three patients in the post-arrest period. A bolus dose of epinephrine may be considered as a treatment for post-arrest hypotension that does not respond to IV fluids, but further studies should be performed prior to routine use.
\end{abstract}

Key words: epinephrine, bolus dose, push dose, pressors, vasopressors, cardiac arrest, hypotension

\section{INTRODUCTION}

Emergency physicians are often faced with very ill patients, a portion of whom undergo cardiac arrest either shortly prior to or in the emergency department. The post-arrest period is a tenuous time in which these patients are at significant risk of re-arrest. Although an exact blood pressure goal has not been fully defined, observational studies have suggested that a post-arrest systolic blood pressure less than $90 \mathrm{~mm} \mathrm{Hg}$ is associated with increased mortality and decreased functional recovery. ${ }^{1-3}$ Additionally, the 2015 American Heart Association guidelines suggest that providers should consider immediately correcting hypotension (defined as a systolic blood pressure less than $90 \mathrm{~mm} \mathrm{Hg}$ or mean arterial blood pressure less than $65 \mathrm{~mm} \mathrm{Hg}$ ) during the post-arrest period. ${ }^{4}$ Most patients will receive intravenous (IV) fluids, followed by vasopressors if they fail to respond to IV fluids. However, IV fluids require time to take effect, and some patients may not be fluid responsive. ${ }^{5,6}$ Additionally, vasopressor infusions require time for obtaining central venous access, as well as mixing the solution and setting up the infusion. This case series describes the use of a bolus dose of epinephrine as a bridge to vasopressor therapy among three patients with significant hypotension after cardiac arrest.

\section{RÉSUMÉ}

L'hypotension artérielle suivant un arrêt cardiaque est associée à des résultats cliniques défavorables. Toutefois, dans une proportion assez importante de cas, les patients peuvent ne pas réagir à l'administration de liquides par voie intraveineuse (i.v.), et les perfusions de vasopresseur nécessitent passablement de temps avant la mise en route. Dans la série de cas décrite ici, il sera question de l'administration, couronnée de succès, d'épinéphrine en bolus afin de traiter rapidement une hypotension réfractaire à l'administration i.v. de liquides chez trois patients, au cours de la période suivant un arrêt cardiaque. L'administration d'épinéphrine en bolus peut être envisagée comme traitement de l'hypotension après un arrêt cardiaque chez les patients ne réagissant pas à l'administration i.v. de liquides, mais il faudrait mener d'autres études avant d'en faire une pratique courante.

\section{CASE REPORT}

\section{Case 1}

A 52-year-old male with a past medical history of hypertension, diabetes, and atrial fibrillation presented by ambulance after a witnessed arrest at home. He received 5 minutes of cardiopulmonary resuscitation (CPR) and $1 \mathrm{mg}$ of epinephrine with return of spontaneous circulation (ROSC). After ROSC was obtained, his heart rate was 42 beats per minute and blood

From the *Department of Emergency Medicine, Rush University Medical Center, Chicago, IL.

Correspondence to: Dr. Michael Gottlieb, Department of Emergency Medicine, Rush University Medical Center, 1750 West Harrison Street, Suite 108, Kellogg, Chicago, IL 60612; Email: michaelgottliebmd@gmail.com 
pressure was $56 / 30 \mathrm{~mm} \mathrm{Hg}$. One liter of IV $0.9 \%$ normal saline was given as a bolus while additional peripheral IV access was obtained. After approximately $500 \mathrm{~mL}$ of IV fluid was delivered, the patient's blood pressure remained as $58 / 28 \mathrm{~mm} \mathrm{Hg}$. The decision was made to place a central venous catheter (CVC) and initiate vasopressors. While the CVC was being placed and the infusion sent from the pharmacy, a bolus dose of epinephrine was given at $1 \mathrm{~mL}$ (10 micrograms) every minute through his peripheral IV line. He received a total of 10 doses. His heart rate improved to an average of 80 beats per minute, and his blood pressure improved to $102 / 60 \mathrm{~mm} \mathrm{Hg}$. After the infusion was initiated, the bolus doses of epinephrine were no longer required. He was subsequently transferred to the intensive care unit. He had an uneventful recovery and was later diagnosed with suspected metoprolol overdose.

\section{Case 2}

A 68-year-old female with a past medical history of throat cancer and recent hospitalization for pneumonia had a witnessed arrest at home. She received immediate CPR from her husband and an additional 10 minutes of CPR from the paramedics before ROSC was obtained. During this time, an advanced airway was placed, intraosseous access was obtained, and two bolus doses of $1 \mathrm{mg}$ of epinephrine were given. Upon arrival to the emergency room, the patient had a heart rate of 108 and blood pressure of $62 / 34 \mathrm{~mm} \mathrm{Hg}$. One liter of $0.9 \%$ normal saline was given through the intraosseous line, while peripheral IV access was obtained. Despite IV fluids, her blood pressure remained low at 72/ $38 \mathrm{mmHg}$. After the peripheral IV line was placed, the patient received $1 \mathrm{~mL}$ (10 micrograms) of a bolus dose of epinephrine every minute (12 total doses) while a central line was placed and norepinephrine infusion was sent from the pharmacy. The blood pressure improved to $106 / 72 \mathrm{~mm} \mathrm{Hg}$ and the patient's vital signs remained stable until the norepinephrine infusion was started. However, prior to a transfer to the intensive care unit, the remainder of the family arrived and requested to withdraw all life-sustaining treatments. The patient was extubated and expired shortly thereafter.

\section{Case 3}

A 71-year-old male with a past medical history of hypertension, diabetes, end-stage renal disease receiving hemodialysis, and left foot osteomyelitis presented in cardiac arrest. CPR was initiated and he received four 1-mg doses of epinephrine, as well as multiple doses of calcium gluconate, sodium bicarbonate, and magnesium based upon concern for a possible hyperkalemic etiology of the arrest. ROSC was obtained. His post-ROSC heart rate was 62 beats per minute and his blood pressure was $64 / 38 \mathrm{~mm} \mathrm{Hg}$. He was given an additional dose of calcium gluconate and sodium bicarbonate, as well as albuterol, insulin, glucose, and antibiotics while the post-arrest labs were in progress. A $500-\mathrm{mL}$ bolus of $0.9 \%$ normal saline was given with no significant change in his blood pressure, so a CVC was placed and a norepinephrine infusion was ordered. While this was being arranged, the bolus dose of epinephrine was given at $1 \mathrm{~mL}$ (10 micrograms) every $1-2$ minutes (8 total doses) with improvement of his blood pressure to 106/ $68 \mathrm{~mm} \mathrm{Hg}$. His post-arrest potassium was 7.8, and he was transferred to the intensive care unit and received emergent hemodialysis. He later had a partial amputation of his left foot, followed by discharge to a skilled nursing facility on long-term antibiotics with no major cognitive deficits noted on a follow-up at 2 weeks.

\section{DISCUSSION}

Maintaining sufficient cardiac perfusion is of major importance during and immediately after cardiac arrest. ${ }^{7}$ Data have suggested that a mean arterial pressure less than $45 \mathrm{~mm} \mathrm{Hg}$ is associated with a significantly increased likelihood of hemodynamic collapse among ill patients ${ }^{8}$ and is associated with increased mortality and decreased functional recovery in patients after cardiac arrest. ${ }^{1-3}$ During the post-arrest period, patients are at high risk of re-arrest, and it is important to ensure sufficient coronary perfusion to reduce this risk. Additionally, many organ systems, including the heart and brain, have been subjected to transient global ischemia during the arrest period, and providers should strive to avoid or minimize any further hypotensionrelated ischemia to prevent additional damage.

Causes of post-arrest hypotension may include hypovolemia, myocardial dysfunction, loss of sympathetic tone, and metabolic acidosis, as well as any continuing complications from the initial etiology of the cardiac arrest. ${ }^{9}$ It is important to attempt to identify the underlying cause(s) prior to initiating treatment when possible. Bedside cardiac ultrasonography can be used to estimate ejection fraction (e.g., E-point septal 
separation and global assessment of contractility) and fluid status (e.g., inferior vena cava size and respiratory changes), while a point-of-care arterial blood gas can assess the metabolic status. The resulting information can allow the provider to target the underlying physiologic cause. For example, hypovolemia is best treated with IV fluids; decreased cardiac contractility may benefit from an inotropic agent (e.g., dobutamine, isoproterenol, epinephrine); loss of sympathetic tone can be treated with a vasopressor (e.g., norepinephrine, epinephrine, dopamine, vasopressin); and acidosis can be addressed with improved end-organ perfusion and a consideration of sodium bicarbonate in some circumstances. Note that epinephrine is both an inotrope and vasopressor, serving as a versatile agent in the management of post-arrest hypotension.

Unfortunately, IV fluids (even using a pressure bag) require time to take effect, and some patients may not respond to IV fluids. ${ }^{5,6}$ Additionally, after the decision is made to initiate inotropic or vasopressor therapy, there is often a delay between when the decision is made and when the patient actually receives the treatment. While it is important to advocate for pre-stocking vasopressors in the emergency department to avoid delays related to obtaining the medication from the pharmacy, there are a number of additional delays, which include obtaining central venous access, pump set up, drug mixing, and medication administration. During this time period, the patient is left with little hemodynamic support.

The theoretical use of a bolus dose of epinephrine has been the subject of frequent discussions in social media. ${ }^{10,11}$ However, to the best of this author's knowledge, this is the first case series discussing the clinical use of a bolus dose of epinephrine in the emergency department to treat refractory hypotension during the post-cardiac arrest period. A review of the existing evidence demonstrates extensive research in the anesthesia literature analysing the use of bolus doses of vasopressors in the operating theatre. Most of the studies have assessed the use of phenylephrine or ephedrine to treat hypotension during elective procedures or after spinal epidural anesthesia among previously healthy pregnant females undergoing a caesarean section, demonstrating improvements in blood pressure and fetal blood gases. ${ }^{12-18}$ There are only a few small studies assessing the use of a single bolus dose of IV epinephrine among previously healthy patients undergoing elective procedures, which have demonstrated resultant increases in blood pressures. ${ }^{19,20}$

However, it is important to note that these are very different patient populations with differing etiologies for their hypotension than the critically ill patients seen in the emergency department and, therefore, they may respond differently to these medications. There was only one recent retrospective analysis of the use of a bolus dose of phenylephrine in the emergency department setting for the treatment of post-intubation hypotension, which demonstrated significant improvements in blood pressure. ${ }^{21}$

Current discussions have suggested that epinephrine may be superior to ephedrine, because the latter is less available, has more potential for incorrect dosing, and has a longer half-life. ${ }^{22,23}$ Epinephrine has also been suggested to avoid the potential reflex bradycardia observed with phenylephrine. ${ }^{22,23}$ However, despite extensive theoretical discussion, there have been no published case reports or studies demonstrating the use of bolus doses of epinephrine in the emergency department setting.

Bolus dose epinephrine is easy to make, using medications already available at the bedside (Figure 1), and may be assembled in less than 20 seconds. The dose is $0.5-2.0 \mathrm{~mL}$ ( $5-20$ micrograms) every minute, titrated to effect, with a time of onset of less than 1 minute and a duration of $5-10$ minutes. This can serve as a valuable bridge while awaiting the effects of IV fluids and the initiation of a vasopressor or inotropic infusion.

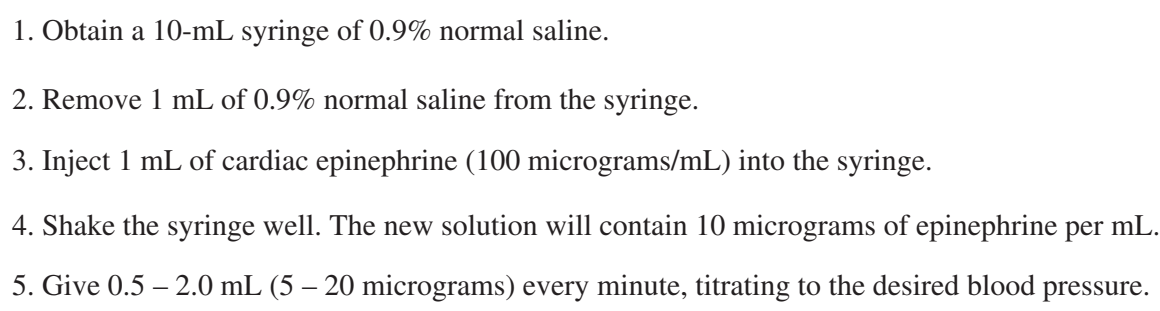

Figure 1. Making a bolus dose of epinephrine. 
Although formal safety trials of this approach are limited, there are several pieces of evidence to suggest the safety of this approach. First, the dose given as a bolus is the same as would be given as an infusion, when divided out per minute. Also, literature suggests that giving vasopressors through a peripheral line is relatively safe and has a very low rate of complications. ${ }^{24}$ Although central access may be safer than peripheral access, most reported complications related to the peripheral administration of vasopressors occurred after use for greater than 35 hours. ${ }^{25}$ Therefore, for very short-term use in the emergency department, the literature suggests that this may be a reasonable route of vasopressor administration while obtaining a central access. One must carefully monitor the extremity for evidence of extravasation while using this technique. However, if it does infiltrate, both phenylephrine and epinephrine have been approved for both subcutaneous and intramuscular use, and the dose of epinephrine is the same as that given as lidocaine with epinephrine for local anesthesia and less than that given for anaphylaxis, providing some support for its theoretical safety.

It is important for the provider to be aware of some potential pitfalls. Providers must be careful to make sure that the medication is mixed appropriately to avoid the potentially dangerous risk of giving a full dose of cardiac epinephrine to the patient. Additionally, providers should avoid bolus doses of epinephrine in the severely tachycardic patient, because the extra beta-adrenergic stimulation may significantly worsen the tachycardia. For these patients, a bolus dose of phenylephrine may be considered as an alternative. Furthermore, one must remember that a bolus dose of epinephrine is a bridge to either clinical improvement or starting a vasopressor infusion, so it is important to reassess and initiate the appropriate next step in management. It is also important not to forget to assess for alternate etiologies for hypotension in these patients and address any treatable causes. Finally, although there is theoretical safety based upon the previous data, formal safety trials have not been performed. There are a number of commonly described techniques to increase the safety of using vasopressors through a peripheral IV line. These include using a larger gauge and a more proximal site when possible; maintaining continuous IV fluids through the line between use; and closely monitoring the extremity and having phentolamine available at the bedside in case of complications.
Future studies should assess this approach in larger samples with special attention to patient-relevant outcomes and safety data, as well as with different vasopressor agents (e.g., norepinephrine).

\section{CONCLUSION}

This case series described the successful use of bolus dose epinephrine to immediately treat refractory hypotension in the post-arrest period. Bolus dose epinephrine may be considered as a treatment for post-arrest hypotension, but further studies should be performed prior to routine use.

Competing interests: None declared.

\section{REFERENCES}

1. Kilgannon JH, Roberts BW, Reihl LR, et al. Early arterial hypotension is common in the post-cardiac arrest syndrome and associated with increased in-hospital mortality. Resuscitation 2008;79(3):410-6.

2. Trzeciak S, Jones AE, Kilgannon JH, et al. Significance of arterial hypotension after resuscitation from cardiac arrest. Crit Care Med 2009;37(11):2895-903; quiz, 2904.

3. Bray JE, Bernard S, Cantwell K, et al. VACAR Steering Committee. The association between systolic blood pressure on arrival at hospital and outcome in adults surviving from out-of-hospital cardiac arrests of presumed cardiac aetiology. Resuscitation 2014;85(4):509-15.

4. Callaway CW, Donnino MW, Fink EL, et al. Part 8: postcardiac arrest care: 2015 American Heart Association guidelines update for cardiopulmonary resuscitation and emergency cardiovascular care. Circulation 2015; 132(18 Suppl 2):S465-82.

5. Marik PE, Cavallazzi R, Vasu T, et al. Dynamic changes in arterial waveform derived variables and fluid responsiveness in mechanically ventilated patients: a systematic review of the literature. Crit Care Med 2009;37(9):2642-7.

6. Marik PE, Monnet X, Teboul JL. Hemodynamic parameters to guide fluid therapy. Ann Intensive Care 2011;1(1):1.

7. Neumar RW, Shuster M, Callaway CW, et al. Part 1: executive summary: 2015 American Heart Association guidelines update for cardiopulmonary resuscitation and emergency cardiovascular care. Circulation 2015; 132(18 Suppl 2):S315-67.

8. Brunauer A, Koköfer A, Bataar O, et al. The arterial blood pressure associated with terminal cardiovascular collapse in critically ill patients: a retrospective cohort study. Crit Care 2014;18(6):719.

9. Peberdy MA, Callaway CW, Neumar RW, et al. Part 9: post-cardiac arrest care: 2010 American Heart Association guidelines for cardiopulmonary resuscitation and emergency cardiovascular care. Circulation 2010;122(18 Suppl 3): S768-86. 
10. Weingart SD. EMCrit Podcast 6 - Push-dose pressors. Available at: http://emcrit.org/podcasts/bolus-dose-pressors/ (accessed 12 April 2016).

11. Browning B. Push-dose pressors. Available at: http://www. emdocs.net/push-dose-pressors/ (accessed 12 April 2016).

12. Lee A, Ngan Kee WD, Gin T. A quantitative, systematic review of randomized controlled trials of ephedrine versus phenylephrine for the management of hypotension during spinal anesthesia for cesarean delivery. Anesth Analg 2002; 94(4):920-6.

13. Ngan Kee WD, Khaw KS, Lau TK, et al. Randomised double-blinded comparison of phenylephrine vs ephedrine for maintaining blood pressure during spinal anaesthesia for nonelective Caesarean section. Anaesthesia 2008;63(12):1319-26.

14. Kol IO, Kaygusuz K, Gursoy S, et al. The effects of intravenous ephedrine during spinal anesthesia for cesarean delivery: a randomized controlled trial. 7 Korea Med Sci 2009;24(5):883-8.

15. Meng L, Cannesson M, Alexander BS, et al. Effect of phenylephrine and ephedrine bolus treatment on cerebral oxygenation in anaesthetized patients. Br 7 Anaesth 2011; 107(2):209-17.

16. Habib AS. A review of the impact of phenylephrine administration on maternal hemodynamics and maternal and neonatal outcomes in women undergoing cesarean delivery under spinal anesthesia. Anesth Analg 2012; 114(2):377-90.

17. Liu H, Huang Y, Diao M, et al. Determination of the $90 \%$ effective dose (ED90) of phenylephrine for hypotension during elective cesarean delivery using a continual reassessment method. Eur 7 Obstet Gynecol Reprod Biol 2015; 194:136-40.

18. Mohta M, Harisinghani P, Sethi AK, et al. Effect of different phenylephrine bolus doses for treatment of hypotension during spinal anaesthesia in patients undergoing elective caesarean section. Anaesth Intensive Care 2015; 43(1):74-80.

19. McCrirrick A, Kestin I. Haemodynamic effects of tracheal compared with intravenous adrenaline. Lancet 1992; 340(8824):868-70.

20. McCrirrick A, Monk CR. Comparison of I.V. and intratracheal administration of adrenaline. Br 7 Anaesth 1994; 72(5):529-32.

21. Panchal AR, Satyanarayan A, Bahadir JD, et al. Efficacy of bolus-dose phenylephrine for peri-intubation hypotension. 7 Emerg Med 2015;49(4):488-94.

22. Selde W.. Push dose epinephrine. A temporizing measure for drugs that have the side-effect of hypotension. $7 E M S$ 2014;39(9):62-3.

23. Weingart S. Push-dose pressors for immediate blood pressure control. Clin Exp Emerg Med 2015;2(2):131-2.

24. Cardenas-Garcia J, Schaub KF, Belchikov YG, et al. Safety of peripheral intravenous administration of vasoactive medication. 7 Hosp Med 2015;10(9):581-5.

25. Loubani OM, Green RS. A systematic review of extravasation and local tissue injury from administration of vasopressors through peripheral intravenous catheters and central venous catheters. 7 Crit Care 2015;30(3):653.e9-17. 